\title{
Identification and characterization of alternative splicing in parasitic nematode transcriptomes
}

\author{
Sahar Abubucker ${ }^{1 \dagger}$, Samantha N McNulty ${ }^{1 \dagger}$, Bruce A Rosa ${ }^{1}$ and Makedonka Mitreva ${ }^{1,2,3^{*}}$
}

\begin{abstract}
Background: Alternative splicing (AS) of mRNA is a vital mechanism for enhancing genomic complexity in eukaryotes. Spliced isoforms of the same gene can have diverse molecular and biological functions and are often differentially expressed across various tissues, times, and conditions. Thus, AS has important implications in the study of parasitic nematodes with complex life cycles. Transcriptomic datasets are available from many species, but data must be revisited with splice-aware assembly protocols to facilitate the study of AS in helminthes.

Methods: We sequenced cDNA from the model worm Caenorhabditis elegans using 454/Roche technology for use as an experimental dataset. Reads were assembled with Newbler software, invoking the cDNA option. Several combinations of parameters were tested and assembled transcripts were verified by comparison with previously reported C. elegans genes and transcript isoforms and with Illumina RNAseq data.

Results: Thoughtful adjustment of program parameters increased the percentage of assembled transcripts that matched known C. elegans sequences, decreased mis-assembly rates (i.e., cis- and trans-chimeras), and improved the coverage of the geneset. The optimized protocol was used to update de novo transcriptome assemblies from nine parasitic nematode species, including important pathogens of humans and domestic animals. Our assemblies indicated AS rates in the range of 20-30\%, typically with 2-3 transcripts per AS locus, depending on the species. Transcript isoforms from the nine species were translated and searched for similarity to known proteins and functional domains. Some 21 InterPro domains, including several involved in nucleotide and chromatin binding, were statistically correlated with AS genetic loci. In most cases, the Roche/454 data explored in this study are the only sequences available from the species in question; however, the recently published genome of the human hookworm Necator americanus provided an additional opportunity to validate our results.
\end{abstract}

Conclusions: Our optimized assembly parameters facilitated the first survey of AS among parasitic nematodes. The nine transcriptome assemblies, their protein translations, and basic annotations are available from Nematode. net as a resource for the research community. These should be useful for studies of specific genes and gene families of interest as well as for curating draft genome assemblies as they become available.

Keywords: Parasitic nematodes, Transcriptomes, Alternative splicing, Next-generation sequencing

\section{Background}

Alternative splicing (AS) is a post-transcriptional, mRNA modification process that allows a single gene to give rise to multiple protein isoforms [1,2]. These spliced isoforms can have distinct molecular functions and biological roles and may be differentially expressed among tissues, life

\footnotetext{
* Correspondence: mmitreva@genome.wustl.edu

${ }^{\dagger}$ Equal contributors

'The Genome Institute, Washington University School of Medicine, 4444

Forest Park Boulevard, St. Louis, MO 63108, USA

2Division of Infectious Diseases, Department of Internal Medicine,

Washington University School of Medicine, St. Louis, MO 63110, USA

Full list of author information is available at the end of the article
}

cycle stages or environmental conditions [3], resulting in involvement in more genetic interactions and biochemical pathways compared to non-AS genes [4]. Therefore, AS provides a significant boost to genomic complexity without necessitating a proportional increase in genome size. AS takes place to some extent in most eukaryotic organisms [5-7], and has been studied extensively in humans and model species, including Caenorhabditis elegans [8-12], but has not received much attention in parasitic nematodes. In fact, information on AS in parasitic nematodes is extremely sparse, and existing

\section{() Biomed Central}

(c) 2014 Abubucker et al.; licensee BioMed Central Ltd. This is an Open Access article distributed under the terms of the Creative Commons Attribution License (http://creativecommons.org/licenses/by/2.0), which permits unrestricted use, distribution, and reproduction in any medium, provided the original work is properly credited. The Creative Commons Public Domain Dedication waiver (http://creativecommons.org/publicdomain/zero/1.0/) applies to the data made available in this article, unless otherwise stated. 
reports have focused on a few or single representative gene(s) [13-16].

High throughput cDNA sequencing is the preferred method for detecting and quantifying AS. Today's most prevalent sequencing protocols (e.g., 454, Illumina, Ion Torrent, etc.) involve fragmentation of nucleic acid molecules, construction of sequencing libraries, and generation of many thousands to millions or even billions of short reads. In the absence of a well-curated genome for comparison, these reads must be re-assembled de novo into contiguous sequences (contigs) that faithfully represent the full-length transcripts from which they were derived. Graph-based assembly algorithms have been developed to maintain associations between transcripts with shared contigs, making it possible to identify different isoforms of the same gene [17]. However, this procedure is computationally challenging, and various studies have shown that de novo cDNA assemblers typically overestimate the number of isoforms associated with a given locus and that many of the predicted isoforms are illegitimate [18-21]. Care must be taken to optimize assembly parameters to minimize errors and maximize accuracy and coverage.

cDNA sequencing is a cost-effective means of gene discovery in non-model organisms, so it often serves as the first line of investigation into an organism's genetic complement. Thus, the transcriptomes of many parasitic nematodes (often including multiple sexes and life cycle stages) have been sequenced and relevant datasets are readily available [22]. Several de novo transcriptome assemblies have been reported [23-30], but most were generated with software that did not account for AS (e.g., Newbler prior to version 2.3, CAP3, etc.). Revisiting existing datasets with a cDNA-specific, splice-aware, assembly protocol would provide a far more accurate impression of AS in parasitic nematodes, a factor that can have important practical implications with respect to pathogenesis, drug susceptibility/resistance, vaccine development, etc. [31,32]. For example, the broad-spectrum anthelmintic drug ivermectin is known to bind tightly to one isoform of the $\alpha 3$ glutamate gated chloride channel subunit but not another, and these isoforms appear to be differentially expressed in susceptible versus resistant strains of the cattle parasites Cooperia oncophora and Ostertagia ostertagi [13,33].

In this study, we used cDNA data from the wellcharacterized model organism Caenorhabditis elegans to define a set of optimized parameters for high-confidence splice isoform prediction using the Newbler assembler. The optimized protocol was then applied to existing and novel cDNA sequences from a diverse array of parasitic nematodes, including Ancylostoma caninum, C. oncophora, Dictyocaulus viviparus, Necator americanus, Oesophagostomum dentatum, Onchocerca flexusoa, O. ostertagi, Teladorsagia circumcincta, and Trichostrongylis colubriformis
[23-30] in the first broad survey of AS among parasitic worms. Our assemblies offer a better impression of genetic and transcriptional complexity in these non-model species and will aid in studies on specific genes/gene families and for annotating and curating draft genomes as they become available.

\section{Methods}

\section{4/Roche library construction, sequencing and data} cleaning

One splice-leader (SL1) and four oligo(dT) cDNA libraries were constructed from DNase treated C. elegans (Bristol N2) RNA according to previously described methods [26]. Libraries were sequenced with a GS 454 FLX pyrosequencer using a standard protocol [34], and raw reads were deposited in the NCBI sequence read archive (SRA) under project number SRP003926. Parasitic nematode sequences were mostly obtained from previous studies, but novel sequences were produced and submitted to the SRA in the same manner (see Additional file 1: Table S1 \& [23-30]).

Raw reads were edited and filtered prior to assembly. Relevant adapter sequences were removed with Cutadapt [35], and reads with an overall quality score less than 20 and an overall dust score less than seven were removed using seq_crumbs software (http://bioinf.comav.upv.es/ seq_crumbs/). The remaining reads were aligned to rRNA $[36,37]$ and bacterial [38] sequence databases with Bowtie2 (version 2.1.0, default parameters [39]) and to the human (hs37) genome and relevant host genomes with Tophat2 (version 2.0.8, default parameters [40]) for contaminant removal. Host genomes, obtained from GenBank, included: Canis lupus famliaris (CanFam3.1) for A. caninum; Bos taurus (Btau4.6.1) for C. oncophora, D. viviparus, and O. ostertagi; Sus scrofa (Sscrofa10.2) for O. dentatum; Ovis aries (Oar3.1) for T. colubriformis and T. circumcincta. O. flexuosa, a parasite of European red deer (Cervus elaphus), and $N$. americanus, maintained in golden hamsters (Mesocricetus auratus), were screened against Bos taurus (Btau4.6.1) and the GenBank rodent database (gbrod, downloaded April 24, 2013), respectively, as close substitutes for the unavailable host genomes.

Cleaned C. elegans Roche/454 reads were mapped to C. elegans coding sequences (WormBase [41] release WS236) with Bowtie2 (version 2.1.0, default parameters [39]) in order to assess the scope of the dataset prior to assembly. The coverage of each feature was assessed using RefCov version 0.3 (http://gmt.genome.wustl.edu/ gmt-refcov/) and coverage was reported in Additional file 2: Table S2.

\section{Assembly and evaluation}

Cleaned, decontaminated C. elegans Roche/454 reads were assembled into isotigs (transcript isoforms) and clustered 
into distinct isogroups (putative genetic loci) using the Newbler assembler (version 2.6, mapasm454_source_ 10142011), invoking the cDNA option. Various combinations of parameters were tested (see Table 1), and the isotigs from each assembly were compared to the C. elegans coding sequences (CDSs) and coding transcripts (CDS + UTRs) included in WormBase [41] release WS236 by BLAST + (version 2.2.27) with a cutoff of $\geq 90 \%$ sequence identity over $\geq 75 \%$ the isotig's length in a single highscoring segment pair. BLAST + was also used to identify chimeric transcripts with non-overlapping, top BLASTN hits to separate chromosomes or BLASTP matches indicating multiple open reading frames coding in opposite directions. Fragmentation (percentage of matched reference genes with multiple, non-overlapping hits) was calculated from WU-BLAST alignments to CDSs using in-house scripts. To further validate our isoform predictions, Illumina RNAseq libraries were generated from C. elegans RNA as previously described [42] (SRA numbers: SRR868958, SRR868932, SRR868957, SRR868939, SRR868942), and the resulting raw reads were mapped to assembled isotigs using Bowtie2 (version 2.1.0, default parameters [39]). The coverage of each isotig, as assessed using RefCov version 0.3 (http://gmt.genome.wustl.edu/ gmt-refcov/), is reported in Additional file 3: Table S3.

Parasitic nematode transcriptomes were assembled with parameters that showed the optimal performance on C. elegans data (minimum overlap of 100 bp and $95 \%$ identity, minimum contig length of 30, and heterozygosity specified). Large or complex datasets were reduced to a manageable size by digital read normalization prior to assembly using khmer with a word size of 31 bp (http:// ged.msu.edu/papers/2012-diginorm/). N. americanus isotigs were compared with transcript isoforms reported along with the genome of $N$. americanus [43] by BLASTN (cutoff of $\geq 90 \%$ sequence identity over $\geq 75 \%$ length of the isotig in a single high-scoring segment pair).

\section{Annotation of parasitic nematode transcriptomes}

Parasite isotigs were searched against the GenBank nonredundant protein database (downloaded July 9, 2013), and non-overlapping top hits with e-value $\leq 1 \mathrm{e}-5$ were recorded (Additional files 4, 5, 6, 7, 8, 9, 10, 11 and 12: Tables S4-S12). Prot4EST [44] was used to generate translations from the parasite isotigs, and InterPro protein domains and gene ontology terms were predicted

Table 1 Caenorhabditis elegans test assemblies ${ }^{1}$

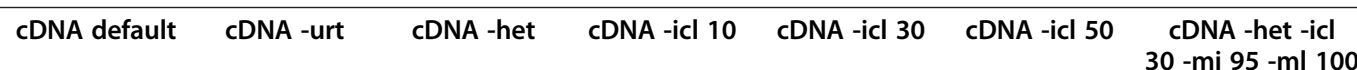

\begin{tabular}{|c|c|c|c|c|c|c|c|}
\hline Assembly statistics & & & & & & & \\
\hline$\%$ Aligned reads & $97.37 \%$ & $99.09 \%$ & $97.34 \%$ & $97.36 \%$ & $97.37 \%$ & $97.37 \%$ & $96.70 \%$ \\
\hline Isotigs & 16737 & 25776 & 16868 & 16548 & 16263 & 16130 & 16772 \\
\hline Isotig N50 & 658 & 563 & 658 & 659 & 662 & 662 & 598 \\
\hline Isogroups & 15403 & 24523 & 15404 & 15401 & 15380 & 15358 & 15940 \\
\hline AS Isogroups & $824(5.35 \%)$ & $823(3.36 \%)$ & $824(5.35 \%)$ & $802(5.21 \%)$ & $741(4.82 \%)$ & $674(4.39 \%)$ & $691(4.34 \%)$ \\
\hline $\begin{array}{l}\text { Ave. isotigs per } \\
\text { AS isogroup }\end{array}$ & 2.62 & 2.52 & 2.78 & 2.43 & 2.19 & 2.15 & 2.20 \\
\hline \multicolumn{8}{|l|}{ Accuracy } \\
\hline fragmentation & $9.40 \%$ & $20.70 \%$ & $9.40 \%$ & $9.40 \%$ & $9.40 \%$ & $9.40 \%$ & $9.90 \%$ \\
\hline trans-chimeric isotigs $^{2}$ & $397(2.37 \%)$ & 407 (1.58\%) & $398(2.36 \%)$ & $398(2.40 \%)$ & 397 (2.44\%) & $385(2.38 \%)$ & $148(0.88 \%)$ \\
\hline cis-chimeric isotigs $^{3}$ & 165 (0.99\%) & $185(0.72 \%)$ & 209 (1.24\%) & $195(1.18 \%)$ & $148(0.91 \%)$ & 145 (0.90\%) & $104(0.62 \%)$ \\
\hline \multicolumn{8}{|l|}{ BLASTN v. CDSs } \\
\hline Isotigs with match ${ }^{4}$ & 6155 (36.77\%) & 10604 (41.14\%) & $6158(36.51 \%)$ & $6083(36.76 \%)$ & $6022(37.03 \%)$ & $5996(37.17 \%)$ & 6385 (38.07\%) \\
\hline Isogroups with match ${ }^{5}$ & 5937 (38.54\%) & $10400(42.41 \%)$ & $5940(38.56 \%)$ & $5933(38.52 \%)$ & $5913(38.45 \%)$ & 5901 (38.42\%) & 6294 (39.49\%) \\
\hline C. elegans genes represented & $5602(27.31 \%)$ & 8470 (41.29\%) & $5604(27.32 \%)$ & $5599(27.29 \%)$ & $5583(27.21 \%)$ & $5579(27.19 \%)$ & $5727(27.92 \%)$ \\
\hline \multicolumn{8}{|l|}{ BLASTN v. CDS + UTR } \\
\hline Isotigs with match ${ }^{4}$ & $11418(68.22 \%)$ & 17031 (66.07\%) & 11456 (67.92\%) & $11217(67.78 \%)$ & $11053(67.96 \%)$ & $10984(68.10 \%)$ & $11778(70.22 \%)$ \\
\hline Isogroups with match ${ }^{5}$ & $10811(70.19 \%)$ & $16512(67.33 \%)$ & $10816(70.22 \%)$ & $10815(70.22 \%)$ & $10789(70.15 \%)$ & $10777(70.17 \%)$ & $11540(72.40 \%)$ \\
\hline C. elegans genes represented & $9600(46.79 \%)$ & $12129(59.12 \%)$ & $9600(46.79 \%)$ & $9598(46.79 \%)$ & $9575(46.67 \%)$ & $9564(46.62 \%)$ & $9748(47.52 \%)$ \\
\hline
\end{tabular}


from translated proteins using InterProScan $[45,46]$. Transcript sequences, peptide translations, and annotations are reported in Additional files 4, 5, 6, 7, 8, 9, 10, 11 and 12: Tables S4-S12 and are available from Nematode.net [22].

\section{Enrichment of AS isogroups associated with functional domains}

The number of alternatively spliced and non-alternatively spliced isogroups associated with each InterPro domain was counted (Additional file 13: Table S13), and a nonparametric binomial distribution test was applied to each InterPro domain to test for enrichment of AS isogroups using the following input parameters: (i) the background frequency of AS isogroups across all species (40.5\%); (ii) the number of AS isogroups associated with the InterPro domain across all species (i.e., number of "successes"); (iii) the total number of isogroups associated with the InterPro domain across all species (i.e., number of "trials"). In order to reduce false positives resulting from poorly represented domains, domains represented by fewer than ten isogroups or fewer than four species were ignored, reducing total number of domains considered from 5,190 to 3,141 (a 39.5\% reduction; Additional file 14: Figure S1). $P$ values calculated for each domain were population corrected using False Discovery Rate (FDR) correction [47], and a significance threshold of 0.01 on the corrected $P$ values was used to determine which InterPro domains were significantly more often associated with AS isogroups than non-AS isogroups.

\section{Results and discussion}

\section{Optimization of assembly parameters}

cDNA libraries were generated from mixed stage $C$. elegans worms and sequenced using Roche/454 technology. Our workflow, from the processing of raw reads to the annotation of transcript isoforms and isogroups, is outlined in Figure 1. After trimming, filtering, and contaminant removal, 1,746,642 high-quality reads were mapped to $C$. elegans CDSs to assess the scope of the dataset prior to assembly, and 8,391 CDS isoforms from 7,487 of the $20,515 C$. elegans genes ( $36.5 \%$ of all genes) showed $\geq 50 \%$ breadth of coverage. This level of coverage is comparable to the level seen in previous transcriptomic surveys of non-model organisms $[26,28,29]$ and is sufficient for testing assembly protocols. It was not our intention to perform a thorough study of AS in C. elegans; studies of this nature have been reported elsewhere [11].

The Newbler assembler, distributed by 454 Life Sciences, is considered the gold standard for Roche/454 read assembly. Using the cDNA option, Newbler identifies regions of shared sequence, termed contigs, and compiles them into full-length transcripts, termed isotigs. Isotigs with shared contigs, theoretically derived from AS of the same gene, are clustered into isogroups representing distinct genetic loci. We tested various combinations of program parameters in order to reduce assembly errors and increase the percentage of isotigs and isogroups that accurately represent known $C$. elegans sequences (Table 1). The best results were obtained with a contig length of $30 \mathrm{bp}$, minimum read overlap of $100 \mathrm{bp}$, minimum sequence identity of $95 \%$ (Table 1, last column). The heterozygous mode had little effect on our C. elegans assemblies, but we chose to invoke this option to accommodate the genetic heterogeneity of our parasitic worm datasets. Using these parameters, 96.7\% of the clean reads were assembled into 15,940 isogroups containing 16,772 isotigs. Some 691 (4.3\%) of these isogroups are associated with more than one isotig, with an average of 2.2 isotigs per AS isogroup (Table 1). Approximately $17 \%$ of the C. elegans genes reported in WormBase build WS236 are associated with more than one CDS isoform with an average of 2.6 isoforms per AS gene [41], and a 2011 study reported that at least $25 \%$ of all C. elegans genes undergo AS [11]. The relatively low rate of AS detected in our test assembly is probably a reflection of the clonal worm population that, despite being mixed-stage, was dominated at the tissue level by the relatively large adult hermaphrodites. Sampling each sex and life cycle stage independently could have provided greater resolution of AS events; however, the aim of this exercise was to optimize assembly protocols, not to explore AS in the model worm.

By adjusting assembly parameters, we were able to increase the number and percentage of isotigs and isogroups that accurately reflected known CDSs, increase the coverage of the gene set, and reduce the rates of misassembled transcripts (i.e., cis- and trans-chimeras). In the best version of our transcriptome assembly, $38.07 \%$ of the isotigs were matched to 7,027 distinct CDS isoforms from 5,727C. elegans genes (Table 1, last column). Match rates increased when isotigs were compared to coding transcripts (CDSs plus untranslated regions) rather than CDSs, indicating that a portion of our sequence data corresponds to untranslated regions at the extreme ends of the transcript.

Despite careful control of assembly parameters, approximately $30 \%$ of the assembled isotigs failed to find a BLAST match to a coding transcript isoform $(\geq 90 \%$ sequence identity over $\geq 75 \%$ of the length of the isotig in a single high-scoring segment pair). The identification of novel AS isoforms and genes is to be expected given that the reported rates of AS in C. elegans have steadily increased over time (Table 2). Therefore, we sought to verify the remaining isotigs using data from another sequencing platform. Of the 4,994 un-matched isotigs, 478 showed $100 \%$ breath of coverage with Illumina RNAseq reads, a strong indication that they reflect real, expressed transcript isoforms, not sequence misassembles (see 


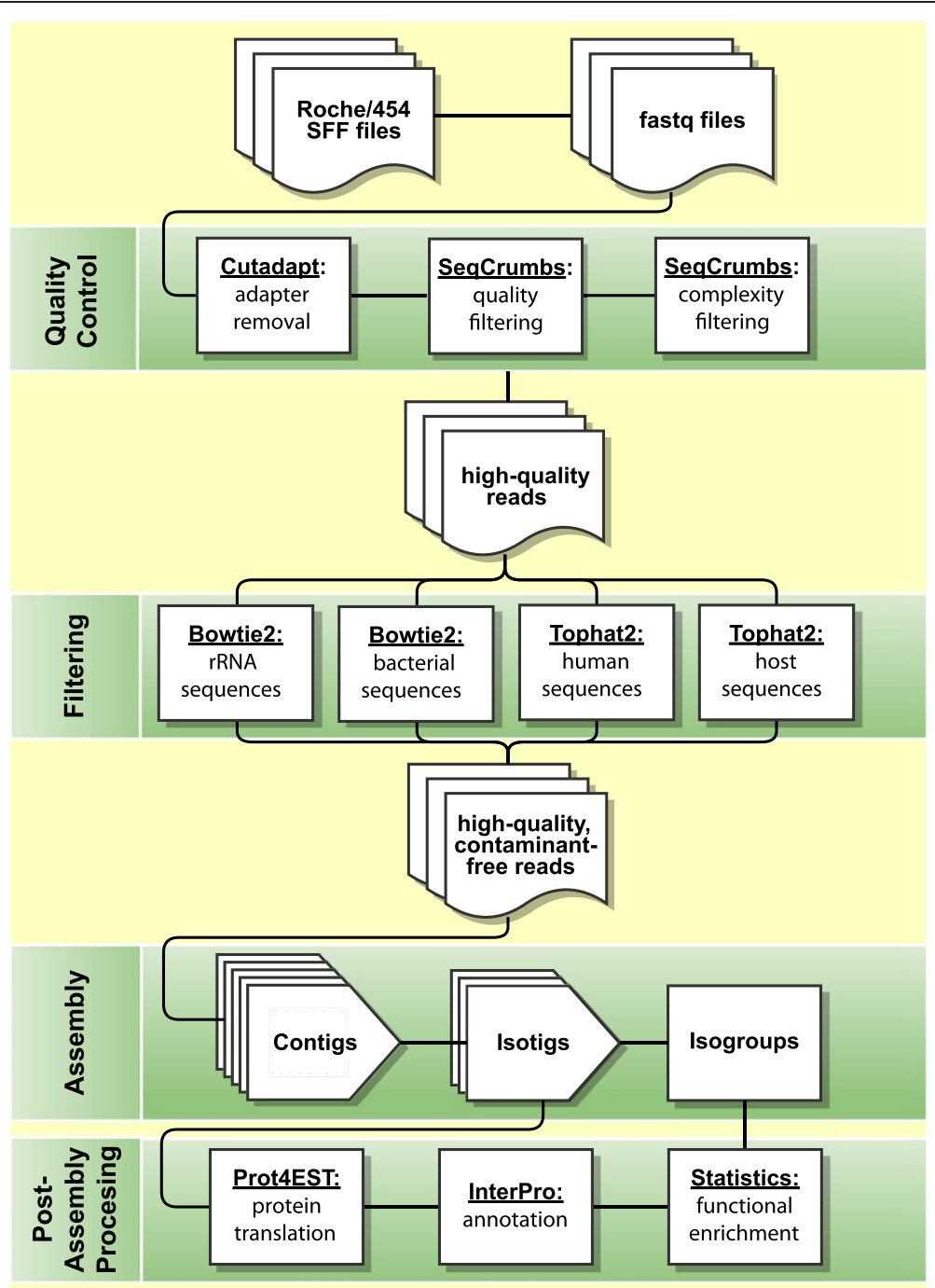

Figure 1 Roche/454 read processing, decontamination, assembly and annotation. Raw Roche/454 reads were converted from sff to fasta format for editing and assembly. Relevant adapter sequences were trimmed, and reads failing to meet quality and complexity thresholds were removed. Reads that successfully map to rRNA, bacterial, human or host sequences were also eliminated. The remaining, high-quality, speciesspecific reads were assembled with Newbler's cDNA specific protocol using our optimized parameter combination, translated using Prot4EST [44], and annotated using InterProScan [45,46]. Statistical analyses can be carried out at the level of isotigs (unique transcripts) or isogroups

(unique genetic loci) depending on the nature of the investigation.

Table 2 Caenorhabditis elegans assembly statistics ${ }^{1}$

\begin{tabular}{lccc}
\hline Build & Date & Gene sequences & Unique CDS isoforms \\
\hline WS150 & Oct 2005 & 20066 & 20066 \\
\hline WS166 & Oct 2006 & 20082 & 23207 \\
\hline WS183 & Oct 2007 & 20155 & 23541 \\
\hline WS196 & Oct 2008 & 20191 & 23902 \\
\hline WS208 & Oct 2009 & 20238 & 24202 \\
\hline WS220 & Oct 2010 & 20405 & 24842 \\
\hline WS228 & Oct 2011 & 20484 & 25391 \\
\hline WS246 & Oct 2012 & 20537 & 26041 \\
\hline WS240 & Oct 2013 & 20538 & 26769
\end{tabular}

${ }^{1}$ Assemblies and annotations from WormBase [41].
Additional file 3: Table S3). An example of this is illustrated in Figure 2. Isogroup00600 contains two distinct isotigs derived from C. elegans gene C18E3.6. One isotig corresponds perfectly to the gene model while the other is missing a 50 base pair segment of the gene's fourth exon. Sequence reads generated on the Roche/454 and Illumina platforms both support the sequence gap despite the fact that this isoform is not represented in WormBase release WS236.

Altogether, 12,265 of the 16,772 isotigs included in our best transcript assembly (73.1\%) were verified either by a match to previously reported transcript isoforms included in WormBase or by our orthologous sequencing 


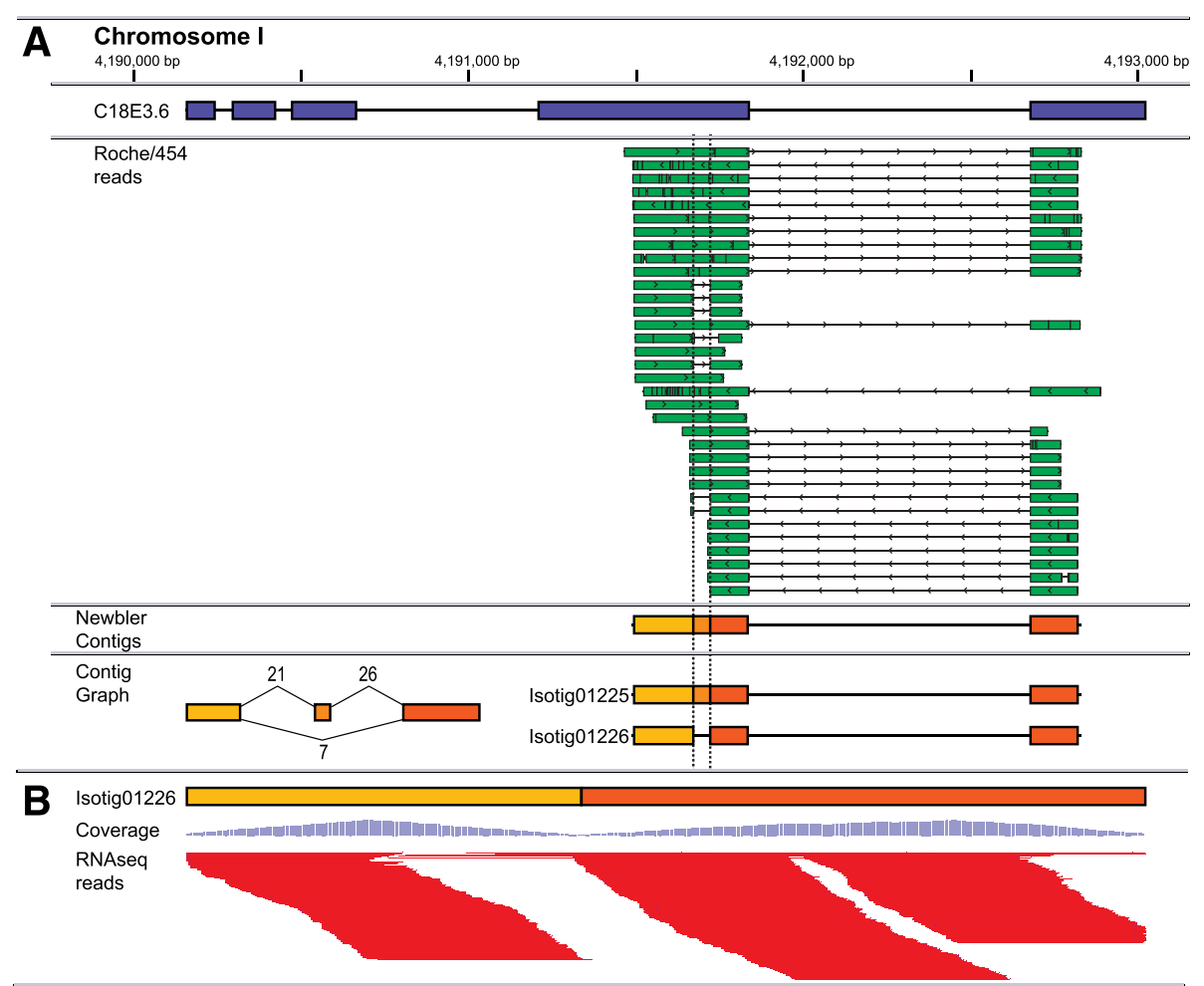

Figure 2 Alternative splicing of C. elegans gene C05B5.5. (A) Isogroup00600 from our de novo cDNA assembly contains two isotigs derived from C. elegans gene C18E3.6 (exons depicted as blue bars in top track). Alignment of Roche/454 reads (green bars with arrowheads indicating directionality) gave rise to three distinct contigs (dark, medium and light orange bars). These contigs were pieced together to form isotigs 01225 and 01226 based on read support displayed in the contig graph. Isotig01225, which contains all three contigs, corresponds perfectly to the gene model (blue bars). However, isotig01226 includes only the first (light orange) and third (dark orange), which results in a 50 bp gap with respect to isotig01225 and the gene model. (B) Illumina RNAseq reads (dark purple, horizontal bars) mapped to isotig01226 further verifies the junction between the first (light orange) and third (dark orange) contigs, with proportional coverage indicated (light purple, vertical bars). This figure was adapted from alignments visualized using the Integrated Genomics Viewer [48,49].

chemistries. Given the limitations presented by today's sequencing technologies and assembly software, no combination of parameters will provide a perfect assembly. Some rate of error is to be expected given the challenges presented by complex, dynamic eukaryotic transcriptomes (e.g., varying expression rates, RNA half-life, secondary structure, AS, etc.). However, the error rates we detect are lower than those reported in other studies (particularly those involving shorter reads and deBruijn graph assemblers $[50,51])$. Clearly, we were able to show improvement over default program parameters using our test dataset, and we expect that the impact of parameter optimization could prove even more vital as the size and complexity of the dataset increases.

The Newbler assembler relies on overlap-layoutconsensus (OLC) algorithms for read assembly. These OLC algorithms may be less likely to overestimate the number of isoforms associated with a given gene compared to de Bruijn graph assemblers [18-20,52], but they are computationally intensive and sensitive to the size and complexity of a dataset. Large datasets with many millions of reads from multiple life cycle stages must be reduced prior to assembly. Our optimized protocol performed well with both randomly down-sampled and digitally normalized read sets (Table 3). Interestingly, digital read normalization eliminated nearly half of the reads without much impact on the quality of the assembly, so we have adopted this as our preferred method for dataset reduction prior to assembly.

\section{Parasitic nematode transcript assemblies}

We re-visited previously published Roche/454 data from C. oncophora [27], O. flexuosa [28], O. ostertagi [27], T. circumcincta [29], and T. colubriformis [25], re-screening and re-assembling with up-to-date, cDNA specific assembly software and our optimized parameters. Additional life cycle stages were sequenced and added to available datasets from $A$. caninum [30], $D$. viviparus [23], N. americanus [26], and O. dentatum [24] prior to assembly (see Additional file 1: Table S1). Together, these nine species represent a diverse array of parasitic nematodes, in terms of biology as well as 
Table 3 Assembly of down-sampled Caenorhabditis elegans read sets

\begin{tabular}{|c|c|c|c|}
\hline & Full & $\begin{array}{c}\text { Random } \\
\text { subset }\end{array}$ & $\begin{array}{c}\text { Normalized } \\
\text { subset }\end{array}$ \\
\hline \multicolumn{4}{|l|}{ Assembly statistics } \\
\hline Reads used & 1746642 & 698656 & 809855 \\
\hline Average read length & 403 & 403 & 383 \\
\hline$\%$ aligned reads & $96.70 \%$ & $94.05 \%$ & $92.68 \%$ \\
\hline Isotigs & 16772 & 12132 & 17322 \\
\hline Isotig N50 & 598 & 569 & 599 \\
\hline Isogroups & 15940 & 11746 & 16129 \\
\hline AS Isogroups & $708(2.65 \%)$ & $341(2.90 \%)$ & $1026(6.36 \%)$ \\
\hline $\begin{array}{l}\text { Average isotigs } \\
\text { per AS isogroup }\end{array}$ & 2.20 & 2.13 & 2.16 \\
\hline \multicolumn{4}{|l|}{ Accuracy } \\
\hline Fragmentation & $9.90 \%$ & $6.30 \%$ & $9.60 \%$ \\
\hline \multicolumn{4}{|l|}{ BLASTN v. CDSs } \\
\hline Isotigs with match ${ }^{1}$ & $6385(38.07 \%)$ & $4424(36.47 \%)$ & $6422(37.07 \%)$ \\
\hline Isogroups with match ${ }^{2}$ & 6294 (39.49\%) & 4380 (37.29\%) & 6224 (38.59\%) \\
\hline $\begin{array}{l}\text { C. elegans genes } \\
\text { matched }\end{array}$ & 5727 (27.92\%) & $4213(20.10 \%)$ & $5628(27.43 \%)$ \\
\hline \multicolumn{4}{|c|}{$\begin{array}{l}\text { Matches were required to meet a cutoff of } \geq 90 \% \text { nucleotide sequence } \\
\text { identity over } \geq 75 \% \text { of the length of the isotigs in a single high-scoring } \\
\text { segment pair. } \\
{ }^{2} \text { Matching isogroups are defined as isogroups containing } \geq 1 \text { isotig matched to } \\
\text { a C. elegans feature. }\end{array}$} \\
\hline
\end{tabular}

phylogeny. Necator americanus, one of the two human hookworms, is thought to infect hundreds of millions of people across the Americas, Sub-Saharan Africa and Asia, and is a leading cause of morbidity in children. A. caninum, the canine hookworm, is an important pathogen in domestic dogs and a model for the study of human hookworm infections. O. flexuosa is a filarial nematode and a close relative of Onchocerca volvulus, the causative agent of African river blindness. O. flexuosa is unique among the Onchocercids in that it is devoid of the bacterial endosymbiont required for development and reproduction in its sister taxa. D. viviparus, the bovine lungworm, is the only Trichostrongylid nematode that resides in the lung during its adulthood. C. oncophora, O. dentatum, O. ostertagi, T. circumcincta, and T. colubriformus are all intestinal worms of livestock animals and are responsible for significant financial losses in the beef, dairy, sheep, goat and pork industries. Projects have been initiated to sequence the genomes of these species (see Table 4 for BioProject ID numbers), but $N$. americanus is the only species for which a draft genome is presently available [43].

Parasitic nematode transcriptome assembly statistics are reported in Table 4. The datasets range in size and complexity from approximately one million reads derived from adult $O$. flexuosa to upwards of 7.5 million reads derived from eggs, larvae and adults of two geographically distinct strains of $O$. ostertagia. As previously discussed, there is a limit to the amount of data that can be processed by OLC assembly algorithms like those implemented by Newbler, so several datasets had to be reduced by digital read normalization prior to assembly (Table 4). Our tests seem to indicate that the complexity of the transcriptome has a greater impact on assembly efficiency than the absolute number of reads. For instance, we were able to assemble some 2.5 million reads from mixed sex adult $T$. colubriformis, whereas a full assembly of the 1.5 million reads derived from L3 and mixed sex adult $N$. americanus was not possible.

The number of isogroups obtained from each assembly ranged from 15,828 from O. flexuosa to 42,785 from the more thoroughly covered transcriptome of $C$. oncophora. Detected rates of $\mathrm{AS}$, as measured by the number of isogroups associated with multiple isotigs, mostly fell within the $20-30 \%$ range, with a maximum AS rate of $34.65 \%$ in D. viviparus (Table 4 ). The AS rates seen in the parasitic nematodes were expected to be similar, as previous studies have shown that splice events are highly conserved among Caenorhabditis species despite hundreds of millions of years of evolutionary separation $[53,54]$. It is also reasonable to expect that the parasitic nematodes, especially those with extremely complex life cycles like $N$. americanus and D. viviparus, would have higher rates of AS than free-living worms like C. elegans due to the increased genomic complexity that may be required to interact with multiple hosts/vectors, host/ vector tissues, and environmental conditions. We did not make an effort to classify or compare the nature of these AS events (e.g., alternative starts and/or stops, intron retention, exon skipping, etc.), but we expect that this will be possible in future studies aimed at exploring AS profiles of particular species in greater detail.

It stands to reason that sampling and sequencing more life cycle stages would lead to increased resolution of AS events. Indeed, including more stages tended to increase the number of isogroups (i.e., genetic loci) identified, but overall AS rates and the average number of isotigs associated with each isogroup remained relatively consistent with the notable exception of $T$. colubriformis. The AS rate reported for $T$. colubriformis (11.68\%) was much lower than AS rates reported for other species represented by a single cDNA library derived from mixed-sex adults $(24.44 \%$ AS in O. flexuosa and $21.14 \%$ AS in T. circumcincta). This disparity may be due to decreased transcriptomic complexity in T. colubriformis, but there may be other explanations. In the case of T. colubriformis, material was obtained from an inbred laboratory strain [25], while O. flexuosa and T. circumcinta material were collected in the field $[28,29]$. O. flexuosa nodules tend to be dominated by large, adult females [55]. Likewise, 
Table 4 Parasitic nematode transcript assemblies

\begin{tabular}{|c|c|c|c|c|c|c|c|c|c|}
\hline & $\begin{array}{l}\text { Ancylostoma } \\
\text { caninum }\end{array}$ & $\begin{array}{l}\text { Cooperia } \\
\text { oncophora }\end{array}$ & $\begin{array}{l}\text { Dictyocaulus } \\
\text { viviparus }\end{array}$ & $\begin{array}{l}\text { Necator } \\
\text { americanus }\end{array}$ & $\begin{array}{c}\text { Oesophagostomum } \\
\text { dentatum }\end{array}$ & $\begin{array}{l}\text { Onchocerca } \\
\text { flexuosa }\end{array}$ & $\begin{array}{c}\text { Ostertagia } \\
\text { ostertagi }\end{array}$ & $\begin{array}{l}\text { Teladorsagia } \\
\text { circumcincta }\end{array}$ & $\begin{array}{l}\text { Trichostrongylus } \\
\text { colubriformis }\end{array}$ \\
\hline Publication & [30] & [27] & [23] & [26] & [24] & {$[28]$} & [27] & [29] & [25] \\
\hline Genome BioProject ID & PRJNA72585 & PRJNA72571 & PRJNA72587 & PRJNA72135 & PRJNA72579 & PRJNA230512 & PRJNA72577 & PRJNA72569 & PRJNA74537 \\
\hline Stages & $\begin{array}{l}\text { Egg, L1, L2, iL3, } \\
\text { aL3, male, female }\end{array}$ & $\begin{array}{l}\text { Egg, L1, L2, i i } 3, \text { a } 3 \text {, } \\
\text { L4, male, female }\end{array}$ & $\begin{array}{l}\text { Egg, L1, iL3, L5, } \\
\text { male, female }\end{array}$ & $\begin{array}{l}\text { iL3, mixed } \\
\text { sex adults }\end{array}$ & $\begin{array}{l}\text { L2, iL3, L4, } \\
\text { male, female }\end{array}$ & $\begin{array}{l}\text { Mixed sex } \\
\text { adults }\end{array}$ & $\begin{array}{l}\text { Egg, L1, L2, iL3, L4, } \\
\text { mixed sex adults }\end{array}$ & $\begin{array}{l}\text { Mixed sex } \\
\text { adults }\end{array}$ & $\begin{array}{c}\text { Mixed } \\
\text { sex adults }\end{array}$ \\
\hline Clean reads & $4,028,728$ & $6,113,083$ & $4,740,349$ & $1,566,641$ & $2,614,527$ & $1,050,204$ & $7,528,633$ & $1,746,999$ & $2,513,840$ \\
\hline Normalized or full assembly & Normalized & Normalized & Normalized & Normalized & Full & Full & Normalized & Full & Full \\
\hline Number of isotigs & 53,978 & 74,506 & 50,581 & 21,320 & 36,795 & 22,728 & 67,599 & 31,065 & 37,640 \\
\hline Average isotig length & $1,029 \mathrm{bp}$ & $763 \mathrm{bp}$ & $964 \mathrm{bp}$ & $866 \mathrm{bp}$ & $815 \mathrm{bp}$ & $820 \mathrm{bp}$ & $889 \mathrm{bp}$ & $989 \mathrm{bp}$ & $535 \mathrm{bp}$ \\
\hline Number of isogroups & 35,422 & 42,785 & 29,960 & 16,233 & 23,061 & 15,828 & 37,189 & 21,780 & 31,546 \\
\hline Number of AS Isogroups & $9,955(28.10 \%)$ & $14,180(33.14 \%)$ & $\begin{array}{c}10,380 \\
(34.65 \%)\end{array}$ & $\begin{array}{c}3,354 \\
(20.66 \%)\end{array}$ & $5,589(24.24 \%)$ & $\begin{array}{c}3,869 \\
(24.44 \%)\end{array}$ & $11,840(31.84 \%)$ & $\begin{array}{c}4,604 \\
(21.14 \%)\end{array}$ & $3,686(11.68 \%)$ \\
\hline Average isotigs per AS isogroup & 2.86 & 3.24 & 2.99 & 2.52 & 3.46 & 2.78 & 3.57 & 3.02 & 2.65 \\
\hline Number of unique translations & 48,713 & 60,697 & 44,784 & 20,286 & 29,478 & 20,436 & 58,022 & 28,041 & 35,669 \\
\hline $\begin{array}{l}\text { Number of unique InterPro } \\
\text { domains }\end{array}$ & 4,103 & 3,967 & 4,110 & 3,823 & 3,978 & 2,212 & 4,903 & 4,550 & 3,454 \\
\hline Number of Unique GO terms & 1,234 & 1,211 & 1,259 & 1,183 & 1,239 & 809 & 1,428 & 1,301 & 1,081 \\
\hline
\end{tabular}


T. circumcinta is a polymorphic species with sex ratios biased towards females [56]. This is significant due to the fact that a patent female represents a broad survey of adult female tissues, embryos in various stages of development, and even stored sperm from males, all of which contribute to diversity in the transcript population. Sequencing additional life cycle stages of $T$. colubriformis or specifically studying adults of other species would provide additional data needed to better understand the obtained results.

The assembled sequences from these nine species, as well as their functional annotations and predicted translations are available from Nematode.net [22] for use by the wider community. Although genome sequencing projects are currently underway, the transcriptomes presented here represent a significant proportion of the sequence data available from these species at this time. These datasets are, therefore, a vital source of information on the genetic content and complexity of these parasites and will remain so even after draft genomes are published as genome sequencing does not, in and of itself, provide any information on AS. Historically, initial reports of draft genomes rarely comment on AS [57-64]. For instance, the draft genome of the well-studied filarial nematode $B$. malayi was published in 2007, but the report made no mention of AS [59]. The most recent dataset available from WormBase (B. malayi WS236) includes multiple isoforms for $16 \%$ of the reported genes, but no comprehensive studies on the subject of AS in B. malayi have been reported despite an abundance of representative RNAseq data [65]. The recently published $N$. americanus genome paper was unique in that it included an estimate of AS based on Illumina RNAseq data generated from L3 and adult worms. Multiple isoforms were identified from approximately $25 \%$ of the 19,151 predicted protein coding genes. Some 1,209 of the 3,354 AS isogroups from $N$. americanus match 1,114 genes reported as AS in the genome study $(\geq 90 \%$ nucleotide sequence identity over $\geq 75 \%$ of the length of the isotigs in a single high-scoring segment pair) [43], while another 65 AS isogroups matched genes that previously lacked evidence for AS. Clearly our assemblies, performed with a special emphasis on AS, will be a useful complement to genome sequencing studies and transcriptome studies performed using orthologous sequencing and assembly approaches.

Table 5 Enrichment of InterPro protein domains among alternatively spliced isogroups

\begin{tabular}{|c|c|c|c|c|c|}
\hline \multicolumn{2}{|c|}{ InterPro protein domain } & \multirow{2}{*}{$\begin{array}{c}\begin{array}{c}\text { Number of } \\
\text { species with } \\
\text { domain }\end{array} \\
9\end{array}$} & \multirow{2}{*}{$\begin{array}{c}\begin{array}{c}\text { Total number of } \\
\text { Isogroups } \\
\text { containing domain }\end{array} \\
858\end{array}$} & \multirow{2}{*}{$\begin{array}{c}\begin{array}{c}\text { Percentage of } \\
\text { isogroups with } \\
\text { domain that are AS* }\end{array} \\
50.7 \%\end{array}$} & \multirow{2}{*}{$\begin{array}{c}\begin{array}{c}\text { P value for } \\
\text { enrichment** }\end{array} \\
2.1 \mathrm{E}-06\end{array}$} \\
\hline IPR000504 & RNA recognition motif domain & & & & \\
\hline IPR016197 & Chromo domain-like & 9 & 128 & $64.1 \%$ & $3.8 \mathrm{E}-05$ \\
\hline IPR012677 & Nucleotide-binding, alpha-beta plait & 9 & 1037 & $48.7 \%$ & $4.2 \mathrm{E}-05$ \\
\hline IPR006092 & Acyl-CoA dehydrogenase, N-terminal & 8 & 53 & $73.6 \%$ & $2.0 \mathrm{E}-04$ \\
\hline IPR013786 & Acyl-CoA dehydrogenase/oxidase, N-terminal & 9 & 68 & $69.1 \%$ & $3.2 \mathrm{E}-04$ \\
\hline IPR003593 & AAA + ATPase domain & 8 & 189 & $57.7 \%$ & 3.6E-04 \\
\hline IPR001412 & Aminoacyl-tRNA synthetase, class I, conserved site & 8 & 43 & $74.4 \%$ & $6.6 \mathrm{E}-04$ \\
\hline IPR006091 & Acyl-CoA oxidase/dehydrogenase, central domain & 9 & 70 & $67.1 \%$ & 7.6E-04 \\
\hline IPR009100 & $\begin{array}{l}\text { Acyl-CoA dehydrogenase/oxidase, N-terminal } \\
\text { and middle domain }\end{array}$ & 9 & 93 & $63.4 \%$ & 8.9E-04 \\
\hline IPR011993 & Pleckstrin homology-like domain & 9 & 474 & $50.4 \%$ & 1.7E-03 \\
\hline IPR014001 & Helicase, superfamily 1/2, ATP-binding domain & 9 & 317 & $52.1 \%$ & 3.7E-03 \\
\hline IPR023780 & Chromo domain & 9 & 79 & $63.3 \%$ & 3.6E-03 \\
\hline IPR000953 & Chromo domain/shadow & 9 & 85 & $62.4 \%$ & 3.7E-03 \\
\hline IPR015421 & $\begin{array}{l}\text { Pyridoxal phosphate-dependent transferase, } \\
\text { major region, subdomain } 1\end{array}$ & 9 & 275 & $52.7 \%$ & $3.8 \mathrm{E}-03$ \\
\hline IPR002194 & Chaperonin TCP-1, conserved site & 8 & 55 & $67.3 \%$ & $3.6 \mathrm{E}-03$ \\
\hline IPR003954 & RNA recognition motif domain, eukaryote & 9 & 41 & $70.7 \%$ & $4.5 \mathrm{E}-03$ \\
\hline IPR006020 & PTB/PI domain & 9 & 71 & $63.4 \%$ & 5.7E-03 \\
\hline IPR001650 & Helicase, C-terminal & 9 & 298 & $51.7 \%$ & $6.8 \mathrm{E}-03$ \\
\hline IPR017998 & Chaperone tailless complex polypeptide 1 (TCP-1) & 9 & 66 & $63.6 \%$ & 7.6E-03 \\
\hline IPR011545 & DNA/RNA helicase, DEAD/DEAH box type, N-terminal & 9 & 275 & $52.0 \%$ & 7.3E-03 \\
\hline IPR002495 & Glycosyl transferase, family 8 & 7 & 11 & $90.9 \%$ & 7.2E-03 \\
\hline
\end{tabular}

*In total, $40.5 \%$ of all isogroups associated with any InterPro domain are AS.

**Binomial test, FDR corrected, threshold value of 0.01 . 


\section{Protein domains associated with alternative splicing}

Given the fact that in AS, both the patterns of splicing as well as the spliced exons themselves, tend to be evolutionarily conserved [53,54], we wanted to explore potential links between AS and genetic function. Coding sequences were predicted for each of the transcript assemblies and these were searched for similarity to InterPro protein domains. A total of 5,692 unique InterPro domains were identified from all species included in this study, with counts ranging from 4,904 domains in $O$. ostertagi to 2,212 in O. flexuosa (Table 4). Some 40.5\% of all isogroups associated with an InterPro domain are AS, and 21 InterPro protein domains were significantly correlated with AS isogroups (Table 5). Functions related to nucleotide binding are prevalent in this list. Nucleic acid binding proteins have a wide variety of functions, localization patterns, and binding preferences that can certainly be affected by AS [66-68]. For example, AS of the UNC-62 transcription factor produces two distinct isoforms in C. elegans. These isoforms localize to different tissues, exhibit different temporal expression patterns, and seem to bind different DNA consensus sequences due to alterations in the DNA binding domain [69,70]. Chromo (CHRomatin Organization Modifier) and chromo-like domains interact with histones and nucleic acids, and studies have shown that AS of these proteins can have major implications on function, which, in turn have implications on gene expression and organismal development [71]. Future studies will be required to further explore the link between AS and protein function in parasitic nematodes, as well as to elucidate its specific biological consequences.

\section{Conclusions}

De novo transcriptome assembly is a complicated procedure that is confounded by varied gene expression patterns, such AS of mRNA. Transcriptome assemblies benefit from the use of optimized parameters designed to increase accurate coverage of the gene set and minimize assembly error. The set of parameters we described was thoroughly tested with $C$. elegans data and verified using well-curated sequences available from WormBase as well as data from an unrelated sequencing chemistry. Our optimized parameters are offered as a guide to assist in the assembly of other nematode transcriptomes, and updated, annotated transcript assemblies from nine species of parasitic worms are offered as a resource to the research community. Rates of AS seem to be similar among the species studied, and 21 InterPro protein domains appear to be enriched among AS transcripts. This represents a first step in exploring AS among parasitic nematodes, an important and relevant topic that should be further investigated in future sequencing studies.

\section{Additional files}

Additional file 1: Table S1. 454/Roche sequencing of parasitic
nematode transcriptomes.

Additional file 2: Table S2. Coverage of Caenorhabditis elegans CDSs with Roche/454 reads.

Additional file 3: Table S3. Coverage of assembled Caenorhabditis elegans isotigs with Illumina RNAseq reads.

Additional file 4: Table S4. The annotated transcriptome of Ancylostoma caninum.

Additional file 5: Table S5. The annotated transcriptome of Cooperia oncophora.

Additional file 6: Table S6. The annotated transcriptome of Dictyocaulus viviparus.

Additional file 7: Table S7. The annotated transcriptome of Necator americanus.

Additional file 8: Table S8. The annotated transcriptome of Oesophagostomum dentatum.

Additional file 9: Table S9. The annotated transcriptome of Onchocerca flexuosa.

Additional file 10: Table S10. The annotated transcriptome of Ostertagia ostertagi.

Additional file 11: Table S11. The annotated transcriptome of Teladorsagia circumcincta.

Additional file 12: Table S12. The annotated transcriptome of Trichostrongylus colubriformis.

Additional file 13: Table S13. Alternatively spliced and non-alternatively spliced isogroups associated with InterPro protein domains.

Additional file 14: Figure S1. Representation of InterPro protein domains among parasitic nematode species and isogroups. The plot indicates the total number of isogroups (from all species) associated and parasitic nematode species associated with a given InterPro protein domain. In order to reduce false positives resulting from poorly represented domains, InterPro domains represented by fewer than ten isogroups and/or fewer than four species were excluded from enrichment analyses. Red lines indicate cutoff values.

Competing interests

The authors declare that they have no competing interests.

\section{Authors' contributions}

SA and MM conceived the study. SA and SNM processed and assembled C. elegans CDNA data and tested the assemblies. SNM processed and assembled parasitic nematode cDNA data and annotated the parasitic nematode transcriptomes. BAR performed statistical analyses. SA, SNM and MM drafted the manuscript. All authors read and approved the final version of the manuscript.

\section{Acknowledgements}

The authors acknowledge The Genome Institute production team for assistance with cDNA/RNA-seq library construction and sequencing and John Martin for providing technical support. This work was supported by $\mathrm{NIH}$ grant to MM.

\section{Author details}

${ }^{1}$ The Genome Institute, Washington University School of Medicine, 4444 Forest Park Boulevard, St. Louis, MO 63108, USA. ²Division of Infectious Diseases, Department of Internal Medicine, Washington University School of Medicine, St. Louis, MO 63110, USA. ²Department of Genetics, Washington University School of Medicine, St. Louis, MO 63108, USA.

Received: 17 January 2014 Accepted: 14 March 2014 Published: 1 April 2014 


\section{References}

1. Breitbart RE, Andreadis A, Nadal-Ginard B: Alternative splicing: a ubiquitous mechanism for the generation of multiple protein isoforms from single genes. Annu Rev Biochem 1987, 56:467-495.

2. Sammeth M, Foissac S, Guigo R: A general definition and nomenclature for alternative splicing events. PLoS Comput Biol 2008, 4:e1000147.

3. Nilsen TW, Graveley BR: Expansion of the eukaryotic proteome by alternative splicing. Nature 2010, 463:457-463.

4. Talavera D, Sheoran R, Lovell SC: Analysis of genetic interaction networks shows that alternatively spliced genes are highly versatile. PLoS One 2013, 8:e55671.

5. Kim E, Magen A, Ast G: Different levels of alternative splicing among eukaryotes. Nucleic Acids Res 2007, 35:125-131.

6. Brett D, Pospisil H, Valcarcel J, Reich J, Bork P: Alternative splicing and genome complexity. Nat Genet 2002, 30:29-30.

7. Irimia M, Rukov JL, Penny D, Roy SW: Functional and evolutionary analysis of alternatively spliced genes is consistent with an early eukaryotic origin of alternative splicing. BMC Evol Biol 2007, 7:188.

8. Zahler AM: Pre-mRNA splicing and its regulation in Caenorhabditis elegans. WormBook 2012:1-21.

9. Venables JP, Tazi J, Juge F: Regulated functional alternative splicing in Drosophila. Nucleic Acids Res 2012, 40:1-10.

10. Pan Q, Shai O, Lee LJ, Frey BJ, Blencowe BJ: Deep surveying of alternative splicing complexity in the human transcriptome by high-throughput sequencing. Nat Genet 2008, 40:1413-1415.

11. Ramani AK, Calarco JA, Pan Q, Mavandadi S, Wang Y, Nelson AC, Lee LJ, Morris Q, Blencowe BJ, Zhen M, Fraser AG: Genome-wide analysis of alternative splicing in Caenorhabditis elegans. Genome Res 2011, 21:342-348.

12. Harrow J, Frankish A, Gonzalez JM, Tapanari E, Diekhans M, Kokocinski F, Aken BL, Barrell D, Zadissa A, Searle S, Barnes I, Bignell A, Boychenko V, Hunt T, Kay M, Mukherjee G, Rajan J, Despacio-Reyes G, Saunders G, Steward C, Harte R, Lin M, Howald C, Tanzer A, Derrien T, Chrast J, Walters N, Balasubramanian S, Pei B, Tress M, et al: GENCODE: the reference human genome annotation for The ENCODE Project. Genome Res 2012, 22:1760-1774

13. El-Abdellati A, De Graef J, Van Zeveren A, Donnan A, Skuce P, Walsh T, Wolstenholme A, Tait A, Vercruysse J, Claerebout E, Geldhof P: Altered avr-14B gene transcription patterns in ivermectin-resistant isolates of the cattle parasites, Cooperia oncophora and Ostertagia ostertagi. Int J Parasitol 2011, 41:951-957.

14. Liebau E, Hoppner J, Muhlmeister M, Burmeister C, Luersen K, Perbandt M, Schmetz C, Buttner D, Brattig N: The secretory omega-class glutathione transferase OvGST3 from the human pathogenic parasite Onchocerca volvulus. FEBS J 2008, 275:3438-3453.

15. Lu SW, Tian D, Borchardt-Wier HB, Wang X: Alternative splicing: a novel mechanism of regulation identified in the chorismate mutase gene of the potato cyst nematode Globodera rostochiensis. Mol Biochem Parasitol 2008, 162:1-15.

16. Massey HC Jr, Ranjit N, Stoltzfus JD, Lok JB: Strongyloides stercoralis daf-2 encodes a divergent ortholog of Caenorhabditis elegans DAF-2. Int $J$ Parasitol 2013, 43:515-520.

17. Miller JR, Koren S, Sutton G: Assembly algorithms for next-generation sequencing data. Genomics 2010, 95:315-327.

18. Clarke K, Yang Y, Marsh R, Xie L, Zhang KK: Comparative analysis of de novo transcriptome assembly. Sci China Life Sci 2013, 56:156-162.

19. Yang Y, Smith SA: Optimizing de novo assembly of short-read RNA-seq data for phylogenomics. BMC Genomics 2013, 14:328.

20. Zhao QY, Wang Y, Kong YM, Luo D, Li X, Hao P: Optimizing de novo transcriptome assembly from short-read RNA-Seq data: a comparative study. BMC Bioinforma 2011, 12(14):S2.

21. Cahais V, Gayral P, Tsagkogeorga G, Melo-Ferreira J, Ballenghien M, Weinert L, Chiari Y, Belkhir K, Ranwez V, Galtier N: Reference-free transcriptome assembly in non-model animals from next-generation sequencing data. Mol Ecol Resour 2012, 12:834-845.

22. Martin J, Abubucker S, Heizer E, Taylor CM, Mitreva M: Nematode.net update 2011: addition of data sets and tools featuring next-generation sequencing data. Nucleic Acids Res 2012, 40:D720-D728.

23. Cantacessi C, Gasser RB, Strube C, Schnieder T, Jex AR, Hall RS, Campbell BE, Young ND, Ranganathan S, Sternberg PW, Mitreva M: Deep insights into Dictyocaulus viviparus transcriptomes provides unique prospects for new drug targets and disease intervention. Biotechnol Adv 2011, 29:261-271.
24. Cantacessi C, Jex AR, Hall RS, Young ND, Campbell BE, Joachim A, Nolan MJ, Abubucker S, Sternberg PW, Ranganathan S, Mitreva M, Gasser RB: A practical, bioinformatic workflow system for large data sets generated by next-generation sequencing. Nucleic Acids Res 2010, 38:e171.

25. Cantacessi C, Mitreva M, Campbell BE, Hall RS, Young ND, Jex AR, Ranganathan S, Gasser RB: First transcriptomic analysis of the economically important parasitic nematode, Trichostrongylus colubriformis, using a next-generation sequencing approach. Infect Genet Evol 2010, 10:1199-1207.

26. Cantacessi C, Mitreva M, Jex AR, Young ND, Campbell BE, Hall RS, Doyle MA, Ralph SA, Rabelo EM, Ranganathan S, Sternberg PW, Loukas A, Gasser RB: Massively parallel sequencing and analysis of the Necator americanus transcriptome. PLoS Negl Trop Dis 2010, 4:e684.

27. Heizer E, Zarlenga DS, Rosa B, Gao X, Gasser RB, De Graef J, Geldhof P, Mitreva M: Transcriptome analyses reveal protein and domain families that delineate stage-related development in the economically important parasitic nematodes. Ostertagia ostertagi and Cooperia oncophora. BMC Genomics 2013, 14:118.

28. McNulty SN, Abubucker S, Simon GM, Mitreva M, McNulty NP, Fischer K, Curtis KC, Brattig NW, Weil GJ, Fischer PU: Transcriptomic and proteomic analyses of a Wolbachia-free filarial parasite provide evidence of trans-kingdom horizontal gene transfer. PLoS One 2012, 7:e45777.

29. Menon R, Gasser RB, Mitreva M, Ranganathan S: An analysis of the transcriptome of Teladorsagia circumcincta: its biological and biotechnological implications. BMC Genomics 2012, 13(7):S10

30. Wang Z, Abubucker S, Martin J, Wilson RK, Hawdon J, Mitreva M: Characterizing Ancylostoma caninum transcriptome and exploring nematode parasitic adaptation. BMC Genomics 2010, 11:307.

31. Bracco L, Kearsey J: The relevance of alternative RNA splicing to pharmacogenomics. Trends Biotechnol 2003, 21:346-353.

32. Hagiwara M: Alternative splicing: a new drug target of the post-genome era. Biochim Biophys Acta 2005, 1754:324-331.

33. Laughton DL, Lunt GG, Wolstenholme AJ: Alternative splicing of a Caenorhabditis elegans gene produces two novel inhibitory amino acid receptor subunits with identical ligand binding domains but different ion channels. Gene 1997, 201:119-125.

34. Margulies M, Egholm M, Altman WE, Attiya S, Bader JS, Bemben LA, Berka J, Braverman MS, Chen YJ, Chen Z, Dewell SB, Du L, Fierro JM, Gomes XV, Godwin BC, He W, Helgesen S, Ho CH, Irzyk GP, Jando SC, Alenquer ML, Jarvie TP, Jirage KB, Kim JB, Knight JR, Lanza JR, Leamon JH, Lefkowitz SM, Lei $\mathrm{M}$, Li J, et al: Genome sequencing in microfabricated high-density picolitre reactors. Nature 2005, 437:376-380.

35. Martin M: Cutadapt removes adapter sequences from high-throughput sequencing reads. EMBnet J 2011, 17:10-12.

36. Pruesse E, Quast C, Knittel K, Fuchs BM, Ludwig W, Peplies J, Glockner FO: SILVA: a comprehensive online resource for quality checked and aligned ribosomal RNA sequence data compatible with ARB. Nucleic Acids Res 2007, 35:7188-7196

37. Quast C, Pruesse E, Yilmaz P, Gerken J, Schweer T, Yarza P, Peplies J, Glockner FO: The SILVA ribosomal RNA gene database project: improved data processing and web-based tools. Nucleic Acids Res 2013, 41:D590-D596.

38. Consortium THM: A framework for human microbiome research. Nature 2012, 486:215-221.

39. Langmead B, Salzberg SL: Fast gapped-read alignment with Bowtie 2 . Nat Methods 2012, 9:357-359.

40. Kim D, Pertea G, Trapnell C, Pimentel H, Kelley R, Salzberg SL: TopHat2: accurate alignment of transcriptomes in the presence of insertions, deletions and gene fusions. Genome Biol 2013, 14:R36.

41. Yook K, Harris TW, Bieri T, Cabunoc A, Chan J, Chen WJ, Davis P, de la Cruz N, Duong A, Fang R, Ganesan U, Grove C, Howe K, Kadam S, Kishore R, Lee R, Li Y, Muller HM, Nakamura C, Nash B, Ozersky P, Paulini M, Raciti D, Rangarajan A, Schindelman G, Shi X, Schwarz EM, Ann Tuli M, Van Auken K, Wang $D$, et al: WormBase 2012: more genomes, more data, new website. Nucleic Acids Res 2012, 40:D735-741.

42. Rosa BA, Jasmer DP, Mitreva M: Genome-Wide Tissue-Specific Gene Expression, Co-expression and Regulation of Co-expressed Genes in Adult Nematode Ascaris suum. PLoS Negl Trop Dis 2014, 8:e2678.

43. Tang YT, Gao X, Rosa BA, Abubucker S, Hallsworth-Pepin K, Martin J, Tyagi R, Heizer E, Zhang X, Bhonagiri-Palsikar V, Minx P, Warren WC, Wang Q, 
Zhan B, Hotez PJ, Sternberg PW, Dougall A, Gaze ST, Mulvenna J, Sotillo J, Ranganathan S, Rabelo EM, Wilson RK, Felgner PL, Bethony J, Hawdon JM, Gasser RB, Loukas A, Mitreva M: Genome of the human hookworm Necator americanus. Nat Genet 2014.

44. Wasmuth J, Blaxter M: Obtaining accurate translations from expressed sequence tags. Methods Mol Biol 2009, 533:221-239.

45. Hunter S, Jones P, Mitchell A, Apweiler R, Attwood TK, Bateman A, Bernard T, Binns D, Bork P, Burge $S$, de Castro E, Coggill P, Corbett M, Das U, Daugherty L, Duquenne L, Finn RD, Fraser M, Gough J, Haft D, Hulo N, Kahn D, Kelly E, Letunic I, Lonsdale D, Lopez R, Madera M, Maslen J, McAnulla C, McDowall J, et al: InterPro in 2011: new developments in the family and domain prediction database. Nucleic Acids Res 2012, 40:D306-D312.

46. Quevillon E, Silventoinen V, Pillai S, Harte N, Mulder N, Apweiler R, Lopez R: InterProScan: protein domains identifier. Nucleic Acids Res 2005, 33:W116-W120.

47. Benjamini $Y$, Hochberg $Y$ : Controlling the false discovery rate: a practical and powerful approach to multiple testing. J R Stat Soc Ser B Methodol 1995, 57:289-300.

48. Thorvaldsdottir H, Robinson JT, Mesirov JP: Integrative Genomics Viewer (IGV): high-performance genomics data visualization and exploration. Brief Bioinform 2013, 14:178-192.

49. Misner I, Bicep C, Lopez P, Halary S, Bapteste E, Lane CE: Sequence comparative analysis using networks: software for evaluating de novo transcript assembly from next-generation sequencing. Mol Biol Evol 2013, 30:1975-1986.

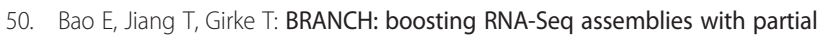
or related genomic sequences. Bioinformatics 2013, 29:1250-1259.

51. Robertson G, Schein J, Chiu R, Corbett R, Field M, Jackman SD, Mungall K, Lee S, Okada HM, Qian JQ, Griffith M, Raymond A, Thiessen N, Cezard T, Butterfield YS, Newsome R, Chan SK, She R, Varhol R, Kamoh B, Prabhu AL, Tam A, Zhao Y, Moore RA, Hirst M, Marra MA, Jones SJ, Hoodless PA, Birol I: De novo assembly and analysis of RNA-seq data. Nat Methods 2010, 7:909-912.

52. Irimia M, Rukov JL, Penny D, Garcia-Fernandez J, Vinther J, Roy SW: Widespread evolutionary conservation of alternatively spliced exons in Caenorhabditis. Mol Biol Evol 2008, 25:375-382.

53. Rukov JL, Irimia M, Mork S, Lund VK, Vinther J, Arctander P: High qualitative and quantitative conservation of alternative splicing in Caenorhabditis elegans and Caenorhabditis briggsae. Mol Biol Evol 2007, 24:909-917.

54. Plenge-Bonig A, Kromer M, Buttner DW: Light and electron microscopy studies on Onchocerca jakutensis and O. flexuosa of red deer show different host-parasite interactions. Parasitol Res 1995, 81:66-73.

55. Craig BH, Pilkington JG, Pemberton JM: Sex ratio and morphological polymorphism in an isolated, endemic Teladorsagia circumcincta population. J Helminthol 2010, 84:208-215.

56. Bai X, Adams BJ, Ciche TA, Clifton S, Gaugler R, Kim KS, Spieth J, Sternberg PW, Wilson RK, Grewal PS: A lover and a fighter: the genome sequence of an entomopathogenic nematode Heterorhabditis bacteriophora. PLoS One 2013, 8:e69618.

57. Desjardins CA, Cerqueira GC, Goldberg JM, Dunning Hotopp JC, Haas BJ, Zucker J, Ribeiro JM, Saif S, Levin JZ, Fan L, Zeng Q, Russ C, Wortman JR, Fink DL, Birren BW, Nutman TB: Genomics of Loa loa, a Wolbachia-free filarial parasite of humans. Nat Genet 2013, 45:495-500.

58. Ghedin E, Wang S, Spiro D, Caler E, Zhao Q, Crabtree J, Allen JE, Delcher AL, Guiliano DB, Miranda-Saavedra D, Angiuoli SV, Creasy T, Amedeo P, Haas B, El-Sayed NM, Wortman JR, Feldblyum T, Tallon L, Schatz M, Shumway M, Koo H, Salzberg SL, Schobel S, Pertea M, Pop M, White O, Barton GJ, Carlow CK, Crawford MJ, Daub J, et al: Draft genome of the filarial nematode parasite Brugia malayi. Science 2007, 317:1756-1760.

59. Godel C, Kumar S, Koutsovoulos G, Ludin P, Nilsson D, Comandatore F, Wrobel N, Thompson M, Schmid CD, Goto S, Bringaud F, Wolstenholme A, Bandi C, Epe C, Kaminsky R, Blaxter M, Maser P: The genome of the heartworm, Dirofilaria immitis, reveals drug and vaccine targets. FASEB J 2012, 26:4650-4661.

60. Jex AR, Liu S, Li B, Young ND, Hall RS, Li Y, Yang L, Zeng N, Xu X, Xiong Z, Chen F, Wu X, Zhang G, Fang X, Kang Y, Anderson GA, Harris TW, Campbell BE, Vlaminck J, Wang T, Cantacessi C, Schwarz EM, Ranganathan S, Geldhof P, Nejsum P, Sternberg PW, Yang H, Wang J, Wang J, Gasser RB: Ascaris suum draft genome. Nature 2011, 479:529-533.

61. Laing R, Kikuchi T, Martinelli A, Tsai IJ, Beech RN, Redman E, Holroyd N, Bartley DJ, Beasley H, Britton C, Curran D, Devaney E, Gilabert A, Hunt M,
Jackson F, Johnston SL, Kryukov I, Li K, Morrison AA, Reid AJ, Sargison N, Saunders GI, Wasmuth JD, Wolstenholme A, Berriman M, Gilleard JS, Cotton JA: The genome and transcriptome of Haemonchus contortus, a key model parasite for drug and vaccine discovery. Genome Biol 2013, 14:R88.

62. Mitreva M, Jasmer DP, Zarlenga DS, Wang Z, Abubucker S, Martin J, Taylor CM, Yin Y, Fulton L, Minx P, Yang SP, Warren WC, Fulton RS, Bhonagiri V, Zhang X, Hallsworth-Pepin K, Clifton SW, McCarter JP, Appleton J, Mardis ER, Wilson RK: The draft genome of the parasitic nematode Trichinella spiralis. Nat Genet 2011, 43:228-235.

63. Schwarz EM, Korhonen PK, Campbell BE, Young ND, Jex AR, Jabbar A, Hall RS, Mondal A, Howe AC, Pell J, Hofmann A, Boag PR, Zhu XQ, Gregory TR, Loukas A, Williams BA, Antoshechkin I, Brown CT, Sternberg PW, Gasser RB: The genome and developmental transcriptome of the strongylid nematode Haemonchus contortus. Genome Biol 2013, 14:R89.

64. Choi YJ, Ghedin E, Berriman M, McQuillan J, Holroyd N, Mayhew GF, Christensen BM, Michalski ML: A deep sequencing approach to comparatively analyze the transcriptome of lifecycle stages of the filarial worm, Brugia malayi. PLoS Negl Trop Dis 2011, 5:e1409.

65. Wollerton MC, Gooding C, Robinson F, Brown EC, Jackson RJ, Smith CW: Differential alternative splicing activity of isoforms of polypyrimidine tract binding protein (PTB). RNA 2001, 7:819-832.

66. MacMorris MA, Zorio DA, Blumenthal T: An exon that prevents transport of a mature mRNA. Proc Natl Acad Sci U S A 1999, 96:3813-3818.

67. Van Nostrand EL, Sanchez-Blanco A, Wu B, Nguyen A, Kim SK: Roles of the developmental regulator unc-62/Homothorax in limiting longevity in Caenorhabditis elegans. PLoS Genet 2013, 9:e1003325.

68. Van Auken K, Weaver D, Robertson B, Sundaram M, Saldi T, Edgar L, Elling U, Lee M, Boese Q, Wood WB: Roles of the Homothorax/Meis/Prep homolog UNC-62 and the Exd/Pbx homologs CEH-20 and CEH-40 in C. elegans embryogenesis. Development 2002, 129:5255-5268.

69. Van Nostrand EL, Kim SK: Integrative analysis of C. elegans modENCODE ChIP-seq data sets to infer gene regulatory interactions. Genome Res 2013, 23:941-953.

70. Boije H, Ring H, Shirazi Fard S, Grundberg I, Nilsson M, Hallbook F: Alternative splicing of the chromodomain protein Morf4l1 pre-mRNA has implications on cell differentiation in the developing chicken retina. J Mol Neurosci 2013, 51:615-628.

71. Kita Y, Nishiyama M, Nakayama Kl: Identification of CHD7S as a novel splicing variant of $\mathrm{CHD} 7$ with functions similar and antagonistic to those of the full-length CHD7L. Genes Cells 2012, 17:536-547.

doi:10.1186/1756-3305-7-151

Cite this article as: Abubucker et al:: Identification and characterization of alternative splicing in parasitic nematode transcriptomes. Parasites \& Vectors 2014 7:151.

\section{Submit your next manuscript to BioMed Central and take full advantage of:}

- Convenient online submission

- Thorough peer review

- No space constraints or color figure charges

- Immediate publication on acceptance

- Inclusion in PubMed, CAS, Scopus and Google Scholar

- Research which is freely available for redistribution 